\title{
Dermatomyositis as the first manifestation of gallbladder adenocarcinoma: case report and literature overview
}

Petra Jurcic

\begin{abstract}
Dermatomyositis (DM) is characterized by pathognomic cutaneous manifestations (heliotrope rash, periorbital edema, Gottron's papules) and proximal muscle weakness. In this paper, I will present the case of a 48-year-old female patient whose dermatomyositis was initially diagnosed as vasculitis. Following the patient's inadequate response to corticosteroid treatment, clinical and radiologic examinations were performed, showing inoperable gallbladder adenocarcinoma. Although initial chemotherapy led to regression, the dermatomyositis developed an independent course with new pathological changes leading to the progression of the disease. I will also present an overview of case reports in English published so far. Gallbladder carcinoma should be added to the list of malignancies with dermatomyositis and has to be excluded by relevant investigation in women.
\end{abstract}

Keywords: Dermatomyositis, Gallbladder, Adenocarcinoma, Paraneoplastic

\section{Background}

Dermatomyositis $(\mathrm{DM})$ is an uncommon inflammatory myopathy characterized by pain and weakness in the proximal muscles and cutaneous manifestations. The skin findings of DM include scaly violaceous papules and plaques overlaying the bony prominences of the hands (Gottron's papules), violaceous patches on the periorbital skin (heliotrope eruption), photodistributed poikilodermatous patches and plaques, scaly plaques on the scalp and lateral thighs, periungual telangiectasia, and ragged cuticles [1]. Malignancies may occur before, simultaneously with or after the onset of DM [2]. The most common malignancies associated with DM are ovarian and lung cancer [3]. The pathogenesis of paraneoplastic DM is still relatively unknown, although there is some evidence to support the involvement of humoral and cellmediated immune systems and the presence of tumour antigens provoking an autoimmune response [3]. Treatment of DM is empirical, often involving a combination of steroids and immunomodulatory drugs [4]. Where $\mathrm{DM}$ is associated with underlying malignancy, tumour therapy may lead to the resolution of DM. Interestingly,

Correspondence: petra.jurcic@gmail.com

Department of Radiation Therapy and Internal Oncology, Tumor Clinic,

Sisters of Charity Hospital, Vinogradska 29, 10000 Zagreb, Croatia a reverse relationship also exists, whereby a flare of DM occurs with tumour recurrence - an association that is demonstrated in this report [5].

\section{Case presentation}

A 48-year-old female patient with history of skull fracture suffered in a traffic accident 25 years ago presented in midJanuary 2014 in the infectology clinic of her local hospital for facial, neck and ear erythema; butterfly-shaped rash; pain in the upper arm muscles; stiffness of hands and dysphagia. Considering the sedimentation (SE) 30 creatine kinase (CK) 308 result, as well as leukocyte (L) 11.4 (white blood cells (WBC) differential percent neutrophils (\% neu) 87, percent lymphocytes (\% lymphs) 7.2, percent monocytes (\% monos) 5.9, percent eosinophils (\% eos) 0 ), the patient was referred to immunology tests (anti-antinuclear antibodies verified by indirect immunofluorescence (ANA IIF) slightly positive and anti-double-strained DNA (antidsDNA), anti-Ro/SSA antibodies (anti-SS-A52), anti-Ro/ SSA antibodies (anti-SS-A60), SSB/La antibody (anti-SSB), anti-Sm antibodies directed against 7 proteins (anti-Sm), autoantibodies directed against the RNP/Sm ribonucleoprotein complex (anti-Sm/RNP), anti-DNA topoisomerase 1 antibody (anti-DNA-topo 1), antibody directed against Jo-1 protein (anti-Jo-1), CENP-B specific anti-centromere 
autoantibodies (anti-CENP-B), autoantibodies to ribosomal P (anti-RIBO P) negative). Since the administered corticosteroid treatment yielded no improvement, a multi-slice computed tomography (MSCT) of the abdomen (Figure 1) was performed, showing a pronounced thickening of the cholecyst wall ranging from 11 to 17 millimeters (mm), scattered and eccentric in appearance; two intraluminally present choleliths, one within the fundus $18 \times 16 \mathrm{~mm}$ and the other infundibular $22 \times 16 \mathrm{~mm}$; and no evident pericholecystic edema. The tumour markers are carcinoembryonic antigen (CEA) $0.7 \mu \mathrm{g} / \mathrm{l}$ and carbohydrate antigen 19-9 (CA 19-9) 964 U/ml. Laparoscopically visualized gallbladder showed malign alterations. Urgent cytological analysis showed malign cells, after which the procedure was reverted to laparotomy which verified omentum carcinomatosis, as well as infiltration of the fifth liver segment. Palpable lymph nodes were in the direction of the hepatoduodenal ligament. The procedure was finished with exploration. Final histopathology report of the omentum was adenocarcinoma (diffuse solid clusters of polymorphous atypical hyperchromatic tumour cells and gland formations with atypical mucous-cylindrical epithelium).

Oncologist suggested treatment with cisplatin $25 \mathrm{mg} / \mathrm{m}^{2}$ (per square meter of body-surface area) and gemcitabine $1,000 \mathrm{mg} / \mathrm{m}^{2}$, each administered on days 1 and 8 and repeated every 21 days for up to 6 cycles, unless disease progression or unacceptable toxicity occurs. Prior to starting chemotherapy in March 2014, the dermatologist increased the corticosteroid dosage (methylprednisolone $60 \mathrm{mg} /$ day) due to newly manifested periorbital edema and macular exanthem in the regions of the trunk, neck and dorsum of the hand (Figures 2 and 3). The patient refused the recommended skin biopsy. Still, there was some improvement in muscular strength.

Prior to the first cycle of chemotherapy, the patient was given two doses of erythrocyte concentrate for normocytic anaemia (haemoglobin (Hgb) $95 \mathrm{~g} / \mathrm{l}$, haematocrit (Ht) $0.345 \mathrm{l} / \mathrm{l}$, mean corpuscular volume (MCV) $82.8 \mathrm{fl}$ ). After 10 days of high-dose corticosteroid therapy, the skin eruptions faded. During and after the second cycle of chemotherapy, the patient complained of general weariness and fatigue.

After 3 cycles of chemotherapy, new skin changes developed on the right upper arm and both thighs, with periorbital edema more pronounced on the left side, despite the $32 \mathrm{mg} /$ day maintenance dose of methylprednisolone. A follow-up MSCT of the abdomen showed a 12-mm thickening of the cholecyst wall - a regression, compared to the initial MSCT result - and increased tumour marker levels (CEA $1.6 \mu \mathrm{g} / \mathrm{l}$, CA 19-9 2,525 U/ml). During the next three chemotherapy cycles, additional skin changes manifested in the armpit and on the scalp; some marker levels also increased (CA 19-9 3,259 U/ml; the gamma-glutamyl transferase (GGT) doubled). Final MSCT of the thorax, abdomen and pelvis was performed after treatment completion in August 2014, showing a progression of hepatic

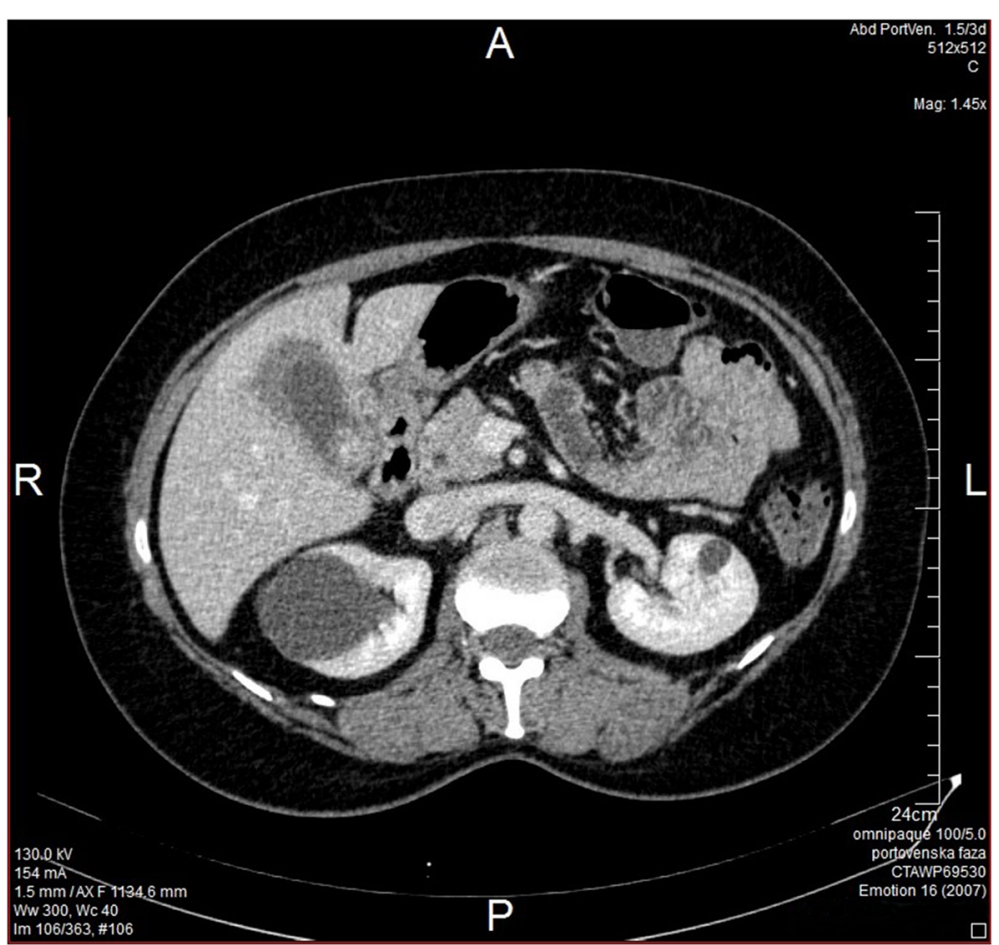

Figure 1 Preoperative MSCT of the abdomen and the pelvis. 


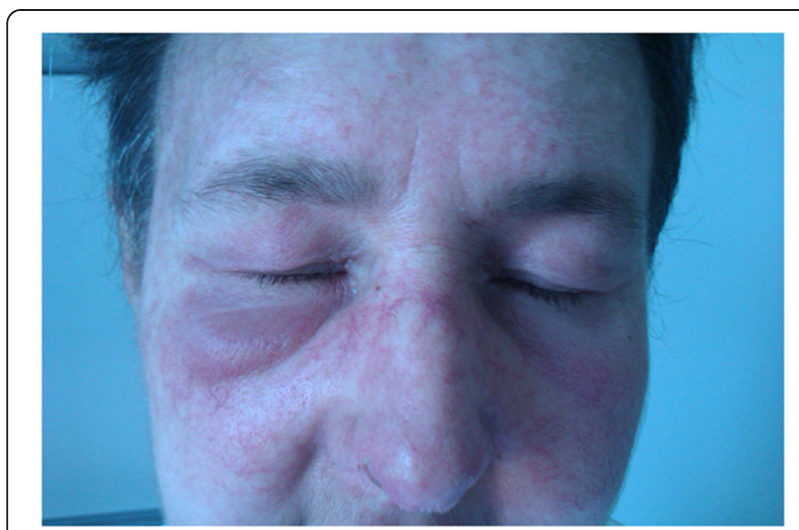

Figure 2 Periorbital edema and macular exanthem of the face.

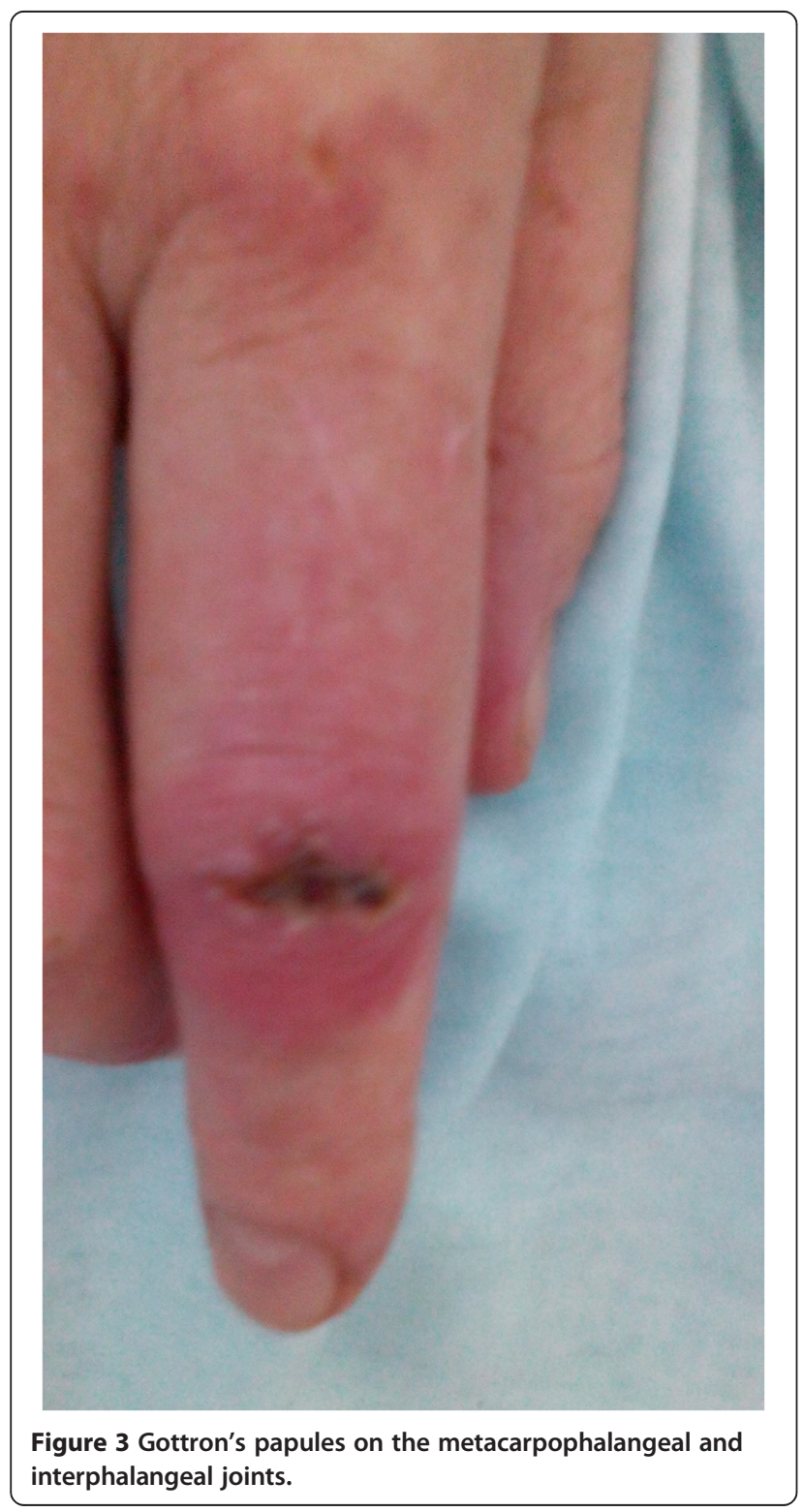

changes and ascites, after which the patient was referred to palliative care.

The patient presented in the case report was treated using the standard care of gall bladder carcinoma. The Helsinki Declaration was obeyed. Ethical board of Hospital Sisters of Charity gave the approval.

\section{Discussion}

Gallbladder carcinoma is the most common and the most malignant tumour of the biliary tract. It occurs mostly between the ages of 60 and 70 and is 2 to 6 times more common in women. Histologically, $95 \%$ of gallbladder carcinoma patients have adenocarcinoma; this was also the case with my patient, as the final histopathology report of the omentum showed. Interestingly, the patient was not aware of her long-standing cholelithiasis and had no pathological condition connected to increased risk.

Gallbladder carcinoma has an extremely bleak prognosis; only $5 \%$ to $10 \%$ of patients who undergo surgery are likely to survive the following 5 years [6]. Also, one third of patients whose surgery is finished with exploration show peritoneal and liver metastases that were not visible on MSCT or MRI. In cases of suspected disseminated malignant disease, diagnostic laparoscopy is recommended [7]. My patient's preoperative CT of the thorax, abdomen and pelvis showed no sign of liver or peritoneal metastases.

Although skin biopsy was not performed on my patient and although she did not test positive for autoantibodies usually present in patients suffering from dermatomyositis, it is my opinion that her clinical presentation is consistent with paraneoplastic dermatomyositis. The patient underwent chemotherapy with cisplatin and gemcitabine, which was in accordance with the results of the study of phase III of patients with advanced or metastatic carcinoma. Adding cisplatin to gemcitabine, that is its interaction between gemcitabine and cisplatin afforded significant progressionfree survival (median, 8.4 versus 6.5 months; hazard ratio (HR), 0.72; 95\% confidence interval (CI), 0.57 to 0.90 ; $p=.003$ ) and overall survival benefits (median, 11.7 versus 8.3 months; HR, 0.70 ; $95 \% \mathrm{CI}, 0.54$ to $0.89 ; p=.002$ ). The most common side effects include decreased WBC count and fatigue [8]. My patient never exhibited decreased WBC count, which was partly due to the effects of corticosteroid therapy. Since receiving the second cycle of chemotherapy, she complained of increased general weariness and fatigue, which could be due to the chemotherapy treatment.

In January 2015, I searched PubMed for terms gallbladder cancer, gallbladder carcinoma and dermatomyositis and found a total of six case reports in English describing dermatomyositis in patients suffering from gallbladder carcinoma [9-13]. All of the cases featured female patients over 44 years of age, some with present risk factors 
(previously verified gallstones). The time interval between the appearance of dermatomyositis and malignant disease ranged from 2 weeks to 2 years. Considering the patient was initially treated in a relatively small facility and the administered treatment did not lead to improvement of her condition, it was soon suspected she had a malignant disease.

In the cases I studied, malignant disease was diagnosed within 2 to 7 months.

My patient initially had elevated CK, slightly positive ANA IIF and negative anti-Jo-1, as described in other patients. The tumour markers were initially determined to be CEA and CA 19-9. CEA was within range, whereas CA 19-9 was 24 times above normal. Elevated serum CEA and CA 19-9 levels could be suggestive of gallbladder cancer, bearing in mind that CA 19-9 has higher specificity, and CEA a higher sensitivity [14]. Although some authors describe a complete regression of skin changes following adequate treatment, in the case of my patient, the dermatomyositis and the malignant disease progressed independently of one another [13]. Following high-dose corticosteroid therapy, the skin changes faded within the same period as described in the case report of another patient; however, after 3 cycles of maintenance therapy, they progressed, although the radiology report verified the regression of the disease.

\section{Conclusions}

Gall bladder carcinoma should be added to the list of malignancies with dermatomyositis and has to be excluded by relevant investigation in women, especially since an inexpensive, simple and easily available initial testing exists - abdomen sonography.

\section{Consent}

Written informed consent was obtained from the patient for publication of this case report and any accompanying images. A copy of the written consent is available for review by the Editor-in-Chief of this journal.

\footnotetext{
Abbreviations

$\%$ eos: percent eosinophils; \% lymphs: percent lymphocytes; \% monos: percent monocytes; \% neu: percent neutrophils; ANA IIF: antinuclear antibodies verified by indirect immunofluorescence; anti-CENP-B: CENP-B specific anti-centromere autoantibodies; anti-DNA-topo 1: anti-DNA topoisomerase 1 antibody; anti-dsDNA: anti-double-strained DNA; anti-Jo-1: antibody directed against Jo-1 protein; anti-RIBO P: autoantibodies to ribosomal P; anti-Sm: anti-Sm antibodies directed against 7 proteins; anti-Sm/RNP: autoantibodies directed against the RNP/Sm ribonucleoprotein complex; anti-SS-A52: anti-Ro/SSA antibodies; anti-SS-A60: anti-Ro/SSA antibodies; anti-SSB: SSB/La antibody;

CA 19-9: carbohydrate antigen 19-9; CEA: carcinoembryonic antigen; Cl: confidence interval; CK: creatine kinase; DM: dermatomyositis; GGT: gamma-glutamyl transferase; Hgb: haemoglobin; HR: hazard ratio; Ht: haematocrit; L: leukocyte; MCV: mean corpuscular volume; mm: millimeter; MSCT: multi-slice computed tomography; SE: sedimentation; WBC: white blood cells.
}

\section{Competing interests}

The author declares no competing interests.

\section{Author's contributions}

PJ wrote the initial draft and composed the manuscript of this paper. The author has read and approved the final manuscript.

\section{Acknowledgements}

I would like to express my sincere gratitude to my PhD mentor, Professor Bozo Kruslin, for his perceptive questions which made me look deeper into the core of the topic. I would also like to thank Mrs. Anica Vrdoljak for technical support and Mrs. Iva Martina Bura for providing language help.

Received: 2 November 2014 Accepted: 7 March 2015

Published online: 27 March 2015

\section{References}

1. Lakhanpal S, Bunch TW, Ilstrup DM, Melton LJ. 3rd polymyositisdermatomyositis and malignant lesions: does an association exist? Mayo Clin Proc. 1986;61:645-53.

2. Zhang W, Jiang SP, Huang L. Dermatomyositis and malignancy: a retrospective study of 115 cases. Eur Rev Med Pharmacol Sci. 2009;13:77-80.

3. Airio A, Pukkala E, Isomaki H. Elevated cancer incidence in patients with dermatomyositis: a population based study. J Rheumatol. 1995;22:1300-3.

4. Wolff K, Johnson R. Fitzpatrick's color atlas and synopsis of clinical dermatology. 6th ed. New York: McGraw-Hill; 2009.

5. Almog Y, Ben-Yehuda A, Ben-Chetrit E. Dermatomyositis associated with the recurrence of transitional cell carcinomas and Kaposi's sarcoma. Clin Exp Rheumatol. 1991:9:285-8.

6. Eckel F, Brunner T, Jelic S, ESMO Guidelines Working Group. Biliary cancer: ESMO Clinical Practice Guidelines for diagnosis, treatment and follow-up. Ann Oncol. 2011;6 Suppl 6:40-4.

7. Goere D, Wagholikar GD, Pessaux P, Carrère N, Sibert A, Vilgrain V, et al. Utility of staging laparoscopy in subsets of biliary cancers: laparoscopy is a powerful diagnostic tool in patients with intrahepatic and gallbladder carcinoma. Surg Endosc. 2006;20:721-5.

8. Valle J, Wasan H, Palmer DH, Cunningham D, Anthoney A, Maraveyas A, et al. Cisplatin plus gemcitabine versus gemcitabine for biliary tract cancer N Engl J Med. 2010;362:1273-81.

9. Yiannopoulos G, Ravazoula P, Meimaris N, Stavropoulos M, Andonopoulos AP. Dermatomyositis in a patient with adenocarcinoma of the gall bladder. Ann Rheum Dis. 2002;61:663-4.

10. Kundu AK, Karmakar PS, Bera AB, Pal SK. Carcinoma of the gall bladder presenting as dermatomyositis. JAPI. 2005;53:219-22.

11. Narasimhaiah DA, Premkumar JA, Moses V, Chacko G. Carcinoma of gall bladder presenting as dermatomyositis. Ann Indian Acad Neurol. 2011;14:44-6.

12. Ni QF, Liu GQ, Pu LY, Kong LL, Kong LB. Dermatomyositis associated with gallbladder carcinoma: a case report. World J Hepatol. 2013;5:230-3.

13. Sawada T, Nakai N, Masuda K, Katoh N. Paraneoplastic dermatomyositis associated with gallbladder carcinoma: a case report and mini-review of the published work. Indian J Dermatol. 2014;59:615-6.

14. Strom BL, Maislin G, West SL, Atkinson B, Herlyn M, Saul S, et al. Serum CEA and CA 19-9: potential future diagnostic or screening tests for gallbladder cancer? Int J Cancer. 1990;45:821-4.

\section{Submit your next manuscript to BioMed Central and take full advantage of:}

- Convenient online submission

- Thorough peer review

- No space constraints or color figure charges

- Immediate publication on acceptance

- Inclusion in PubMed, CAS, Scopus and Google Scholar

- Research which is freely available for redistribution 